\title{
Bar properties as seen in the Spitzer Survey of Stellar Structure in Galaxies
}

\author{
Kartik Sheth ${ }^{1}$ and The Spitzer Survey for Stellar Structure in \\ Galaxies $\left(\mathbf{S}^{4} \mathbf{G}\right)$ Team $^{2}$
}

${ }^{1}$ National Radio Astronomy Observatory United States, email: astrokartik@gmail.com

${ }^{2}$ Various Institutions world-wide

\begin{abstract}
Bars serve a crucial signpost in galaxy evolution because they form quickly once a disk is sufficiently massive and dynamically cold. Although the bar fraction in the local Universe is well-established since the mid-60s, a variety of studies have concluded varying bar fractions due to different definitions of bars, use of low quality data or different sample selection. The Spitzer Survey of Stellar Structure in Galaxies $\left(S^{4} \mathrm{G}\right)$ offers us the ideal data set for resolving this outstanding issue once and for all. $\mathrm{S}^{4} \mathrm{G}$ consists of over 2000 nearby galaxies chosen based on optical brightness, distance, galactic latitude and size in a $40 \mathrm{Mpc}$ volume. With a 4 minute integration time per pixel over $>1.5 \times \mathrm{D} 25$ diameter for each galaxy, the data provide the deepest, homogenous, mid-infrared (3.6 and 4.5 microns) data on the nearby Universe. The data are so deep that we are tracing stellar surface densities $<<1$ solar mass per square parsec. With these data we can confidently constrain the bar fraction and thus shed important light on the evolutionary state of galaxies as a function of mass, environment and other galaxy host properties.
\end{abstract}

